\title{
Rising Rates of Syphilis in the Era of Syphilis Elimination
}

\author{
ROXANNE P. KERANI, PHD, ${ }^{\star} \dagger$ H. HUNTER HANDSFIELD, MD, ${ }^{\ddagger} \ddagger$ MARK S. STENGER, MS, $\S$ TARANEH SHAFII, MD, ${ }^{\star} \dagger$ \\ ELLEN ZICK, BA, $\dagger$ DEVON BREWER, PHD,\| AND MATTHEW R. GOLDEN, MD, MPH*†
}

\begin{abstract}
Objective: The objective of this study was to assess the impact of syphilis control activities in King County, Washington.

Study Design: We calculated rates of early syphilis and trends in numbers of persons tested and diagnosed through screening and partner notification from 1998 to 2005.

Results: Early syphilis cases increased from 38 in 1998 to 188 in 2005 with $92 \%$ occurring among men who have sex with men (MSM). Our health department conducted public awareness campaigns, increased publicly financed syphilis screening among MSM by $179 \%$, and intensified partner notification efforts. Despite these efforts, the prevalence of syphilis among screened populations was only $1.1 \%$, and $\mathbf{7 1 \%}$ syphilis cases were diagnosed after seeking care for symptoms. The proportion of cases diagnosed through screening and partner notification did not significantly change during the evaluation period. Early syphilis incidence among MSM more than doubled between 2003 and 2005.
\end{abstract}

Conclusions: New, innovative approaches to syphilis control are needed.

IN 1996, LOCALLY TRANSMITTED SYPHILIS was eliminated from King County, Washington. ${ }^{1}$ The next year, syphilis was reintroduced, and the previously observed pattern of high rates of syphilis among heterosexuals in association with crack cocaine use and commercial sex work was replaced by one in which men who have sex with men (MSM) accounted for the majority of cases. ${ }^{2}$

The National Plan to Eliminate Syphilis (NPES) was introduced by the Centers for Disease Control and Prevention (CDC) in 1999, and in 2001, King County was selected as one of several highmorbidity areas designated to receive funding for syphilis elimination activities. Since then, King County has used this NPES funding to strengthen its efforts in the areas of syphilis surveillance, community involvement, rapid outbreak response, expanded clinical and laboratory services, and enhanced health promotion. These activities constitute the core strategies identified by the NPES as critical in the effort to control syphilis. ${ }^{3}$ Although not identified as a core strategy in the NPES, partner notification has also been advocated as a key component in eliminating syphilis, and King County has strived to improve partner notification services for syphilis as well.

Despite these efforts, the syphilis epidemic is ongoing in King County and, in fact, accelerated through 2005. We undertook the current study to examine trends in rates of early syphilis since the

The authors gratefully acknowledge the assistance and support of Barbara Krekeler, Keith Okita, Fred Koch, Rolf Pedersen, Michelle Perry, the disease intervention specialists, and other staff of the Public Health-Seattle and King County STD Control Program.

Correspondence: Roxanne P. Kerani, PhD, Public Health-Seattle \& King County STD Program, Harborview Medical Center, Box 359777, 325 9th Avenue, Seattle, WA 98104-2499. E-mail: rkerani@u.washington.edu

Received for publication January 18, 2006, and accepted May 29, 2006.
From the *University of Washington, Seattle, Washington; †Public Health-Seattle \& King County, Seattle, Washington; the ¥Battelle Center for Public Health Research and Evaluation, Seattle, Washington; \$Washington State Department of Health, Olympia, Washington; and |Interdisciplinary Scientific Research, Seattle, Washington

current epidemic began and to evaluate the impact of our current syphilis control strategies.

\section{Materials and Methods}

Setting

The study was carried out in King County, Washington, which encompasses Seattle and much of the surrounding metropolitan area. In 2005, King County had an estimated population size of 1,793,583 (http://factfinder.census.gov/home/saff).

\section{Data Sources}

Laboratories and medical providers are required by Washington State law to report cases of syphilis diagnosed in King County to Public Health-Seattle \& King County (PHSKC). Specimens testing rapid plasma reagin (RPR) -positive in public or private laboratories in King County are sent to PHSKC for confirmatory testing using Treponema pallidum particle agglutination tests (TPPA). No syphilis confirmatory testing is conducted by other laboratories in the county, assuring referral of all RPR-positive specimens and virtually $100 \%$ ascertainment of serologically diagnosed syphilis cases in King County. Cases occurring in persons $>60$ years of age with a positive TPPA and RPR titer $\leq 1: 4$ are considered to represent latent, noninfectious (often previously treated) cases and are administratively closed. Cases not meeting these closure criteria are further investigated typically after first contacting the patient's healthcare provider to ascertain the clinical stage of disease. All early syphilis cases (primary, secondary, and early latent syphilis) are interviewed by PHSKC disease intervention specialists (DIS) regarding history of symptoms, demographics, risk behaviors, and partner contact information for case finding and partner notification. We used standard CDC contact periods for primary, secondary, and early latent syphilis as the time period of interest when interviewing patients regarding their sex partners and risk behaviors. ${ }^{4}$

Our primary study period was 1998 to 2005, the time during which an epidemic of syphilis among King County MSM developed and later escalated. Although most of our analyses are for this time period, some data were not consistently collected until later in the study period, and thus some of our analyses are limited to the period 2003 to 2005. Annual incidence from 1998 through 2005 was calculated from case report data, estimates of the King County population prepared by the Washington State Office of Financial Management 


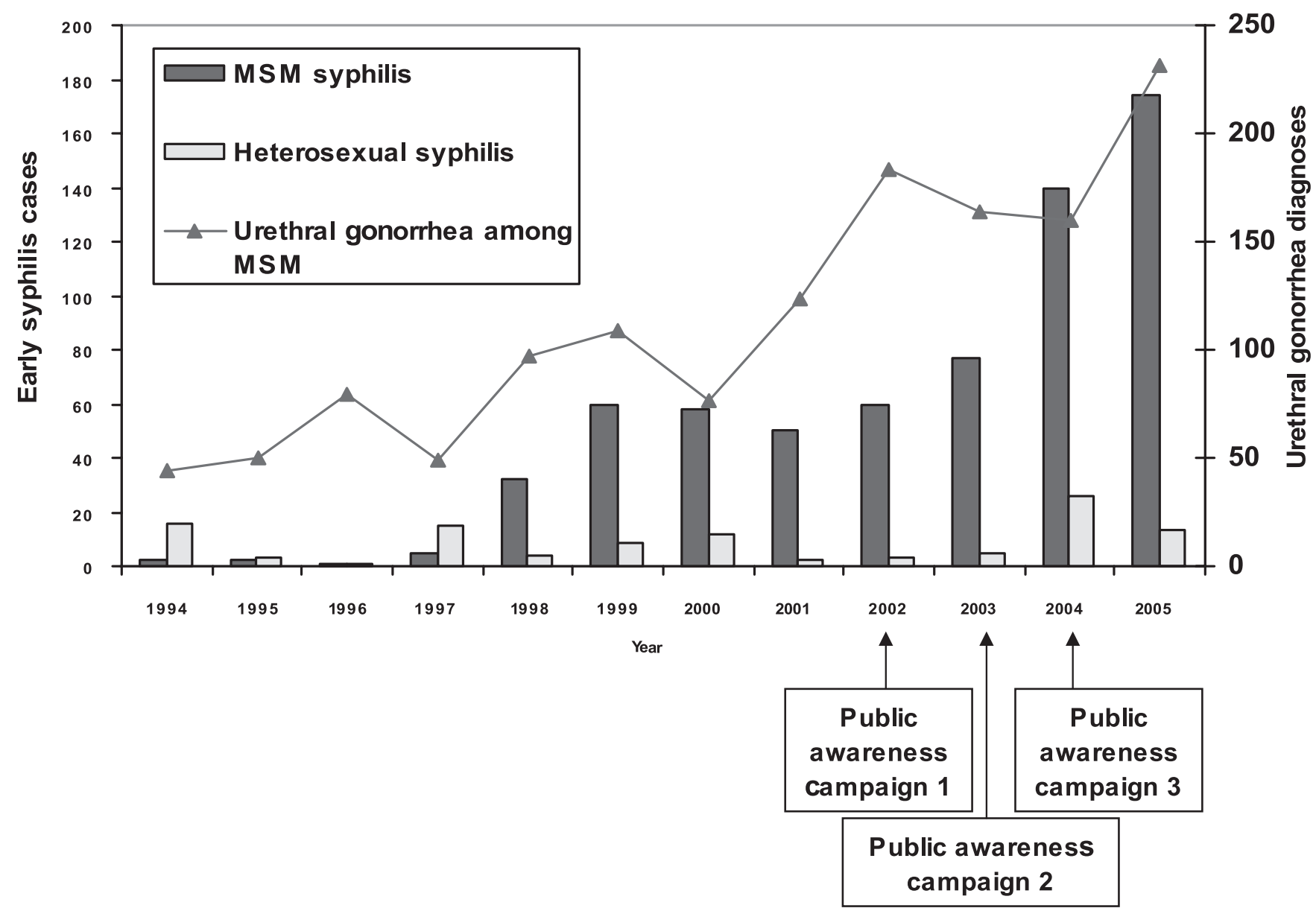

Fig. 1. Early syphilis among King County men who have sex with men (MSM) and heterosexuals, with MSM urethral gonorrhea diagnoses from the Public Health-Seattle \& King County Sexually Transmitted Disease Clinic, 1994-2005.

for intercensal years, and U.S. Census population data for 2000. In addition to overall incidence, we calculated incidence among MSM and heterosexuals. Historical estimates for the MSM population size were derived from 1990 U.S. Census data and published reports of the proportion of all men who are sexually active $\mathrm{MSM}^{5}$; the range of earlier estimates corresponds well with later published estimates of the MSM population size. ${ }^{6}$ The PHSKC HIV/AIDS Epidemiology Unit provided these estimates as well as estimates of the size of the HIV-infected population. ${ }^{6,7}$

We assessed MSM's perceptions of the syphilis problem and sexually transmitted disease (STD) testing experience using results from a random digit dial study conducted among King County residents of zip codes with large MSM populations between February and May 2003. Methods associated with this survey have been previously described. ${ }^{8}$

\section{Analyses}

We used Fisher's Exact test to compare categorical variables and Wilcoxon rank sum test to examine differences in the number of sexual partners reported by MSM and heterosexual cases. Reason for visit data were taken directly from the case report form filled out by the reporting providers. Although providers are instructed to indicate one reason for examination and their potential choices are "symptomatic," "routine examination-no symptoms," and "exposed to infection," providers may be unaware that patients had a previous positive screening test or were contacts to syphilis. Additionally, patients may also be both contacts to syphilis and symptomatic. To minimize the bias potentially introduced by these issues, we compared reason for examination from case report forms with disease investigation and field records for the study period, and in a small number of cases, we reclassified the reason for the examination based on this additional information.

Partner management measures were based on data collected through DIS documenting the outcome of field investigations and included the contact index (number of sex partners identified per case), the epidemiologic index (number of sex partners preventively treated per case), and the brought-to-treatment index (number of new early syphilis cases identified through partner notification per case). We also calculated the proportion of positive treponemal tests among asymptomatic individuals with no history of syphilis for all local screening venues for the time period 1998 to 2005. All analyses were carried out with SAS (SAS Institute Inc., Cary, NC).

\section{Results}

Trends and Epidemiologic Characteristics of Syphilis in King County

From 1998 to 2005, newly diagnosed cases of early syphilis were reported in 731 King County residents. Of these, 706 $(97 \%)$ were men, of whom $651(92 \%)$ were MSM. Overall, 158 


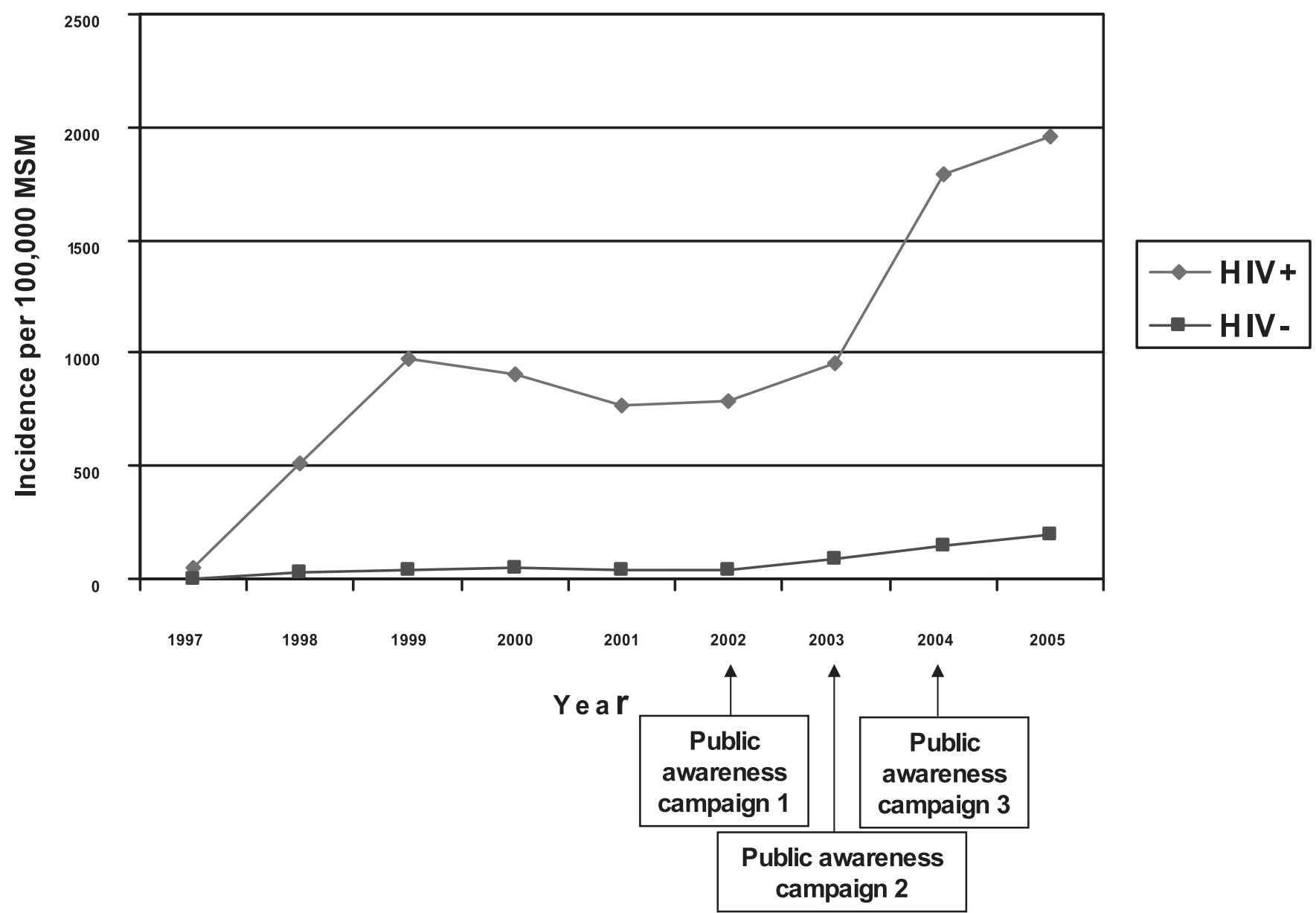

Fig. 2. Incidence of early syphilis among men who have sex with men (MSM) by HIV status, King County, Washington, $1998-2005$.

$(22 \%)$ of the total cases were staged as primary, $399(55 \%)$ as secondary, and $174(24 \%)$ as early latent syphilis. The percentage of cases with primary or secondary $(\mathrm{P} \& S)$ has decreased slightly over time ranging from $84.2 \%$ in 1998 to $71.3 \%$ in 2005. There was no significant difference in the distribution of syphilis stage among heterosexual men, MSM, and women. Three hundred seventy-seven $(57.9 \%)$ of 651 MSM diagnosed with early syphilis were HIV-positive.

Early syphilis among MSM increased from one case in 1996 to 77 cases in 2003 and 174 in 2005, an increase of $125 \%$ from 2003 to 2005 (Fig. 1). Concurrent with this rise in syphilis cases in MSM, the number of cases of symptomatic gonococcal urethritis in MSM seen in the PHSKC STD Clinic annually more than doubled, from 79 cases in 1996 to 231 in 2005. Cases of early syphilis in women and heterosexual men were stable from 1994 to 2003 with a mean of 7 cases annually. There was a substantial increase in heterosexual cases to 26 cases in 2004 and a subsequent decrease to 13 cases in 2005 (Fig. 1).

We compared early syphilis cases in MSM and heterosexuals for 2004 when substantial numbers of cases occurred in women and heterosexual men. Among MSM, 78 (56\%) of 139 cases were HIV-positive compared with one (4\%) of 26 women and heterosexual men $(P<0.0001)$. Sixty-eight percent of MSM, compared with $35 \%$ of heterosexuals, reported anonymous sex partners during the infectious period $(P=0.002)$, and $27 \%$ of MSM and no heterosexuals reported using the Internet to find sex partners
$(P<0.0001)$. Among heterosexual cases, $62 \%$ were sex workers or acknowledged sex with a sex worker compared with 2 MSM $(1 \%)(P<0.0001)$. MSM reported a median of 5 total and 2 anonymous sex partners during the infectious period, whereas heterosexuals reported a median of 3 total and zero anonymous partners $(P<0.008$ and $P=0.013$, respectively).

Figure 2 illustrates the course of the early syphilis epidemic in MSM according to HIV status. The estimated incidence of syphilis among HIV-negative MSM rose 385\% from 2002 to 2004, from 41 to 199 cases per 100,000 MSM. The estimated rate of early syphilis among HIV-positive MSM was substantially higher but was relatively stable from 1999 to 2003 with rates of 767 to 975 per 100,000 . This rate rose sharply in 2004 to 1,788 cases per 100,000 and increased again in 2005 to 1,969 per 100,000 . The proportion of MSM with early syphilis who were HIV-positive decreased from $69 \%$ in 1998 to $50 \%$ in 2005.

\section{Public Health-Seattle \& King County's Response to Syphilis}

To increase awareness of the syphilis epidemic, we conducted 3 public awareness campaigns on syphilis prevention among MSM from 2002 through 2004. All 3 campaigns promoted syphilis testing through posters and advertisements in a variety of media, including bus banners and print media with high MSM readership such as Seattle Gay News. A component of the 2002 campaign was creation of the MSM Task Force comprised of community leaders, 
TABLE 1. Prevalence of Syphilis in Screened Populations, ${ }^{*}$ King County, Washington, 1998-2005

\begin{tabular}{llrrr}
\hline Screening Venue & \multicolumn{1}{c}{$\begin{array}{c}\text { Primary } \\
\text { Population }\end{array}$} & No. of Tests & $\begin{array}{c}\text { Positive } \\
\text { Tests }\end{array}$ & Prevalence \\
\hline Sexually transmitted disease clinic & MSM & 30,158 & 321 & $1.1 \%$ \\
Bathhouses/sex clubs ${ }^{\dagger}$ & MSM & 1,521 & 20 & $1.3 \%$ \\
Community-based organizations & MSM & 2,143 & 18 & $0.8 \%$ \\
Jails & Heterosexuals & 3,387 & 16 & $0.4 \%$ \\
Peer referral program & Heterosexuals & 238 & 2 & $0.8 \%$ \\
Total & & 37,447 & 400 & $1.1 \%$ \\
\hline
\end{tabular}

*Includes screening among asymptomatic individuals reporting no history of syphilis.

†Syphilis screening in bathhouses and sex clubs includes anonymous tests and may include latent syphilis cases.

MSM indicates men who have sex with men.

representatives of community-based organizations, and public health staff. The Task Force continues to meet regularly to monitor important health issues in the MSM community, generate suggestions for prevention, and provide feedback to public health. A random digit dial study of 400 MSM conducted in $2003^{8}$ found that $53 \%$ of MSM believed that STDs were a bigger problem among MSM than they were 5 years earlier. Only 3\% of MSM believed that STDs were less of a problem than they were 5 years earlier ( $45 \%$ believed that STDs "were about the same"). Overall, $46 \%$ of MSM reported being tested for STDs in the preceding year, including $58 \%$ of the 218 men who acknowledged having more than one sex partner and $70 \%$ of the 120 men who reported having $\geq 5$ in the preceding year.
As the local syphilis epidemic progressed, the number of annual syphilis tests performed by PHSKC, including tests in the STD Clinic, county jails, community-based organizations, bathhouses, and sex clubs, grew $47 \%$, from 7,717 tests in 1998 to 11,373 in 2005. The number of tests performed in venues specifically targeting MSM rose $179 \%$, from 1,575 tests in 1998 to 4,395 in 2005 . Table 1 displays the prevalence of confirmed positive serologic tests among asymptomatic individuals with no history of syphilis in a variety of local screening venues. Screening yields ranged from $0.4 \%$ in local jails to $1.3 \%$ in bathhouses and sex clubs.

Concurrent with increases in syphilis incidence and the number of syphilis tests performed, increased emphasis was placed on partner notification activities. Figure 3 shows that the contact

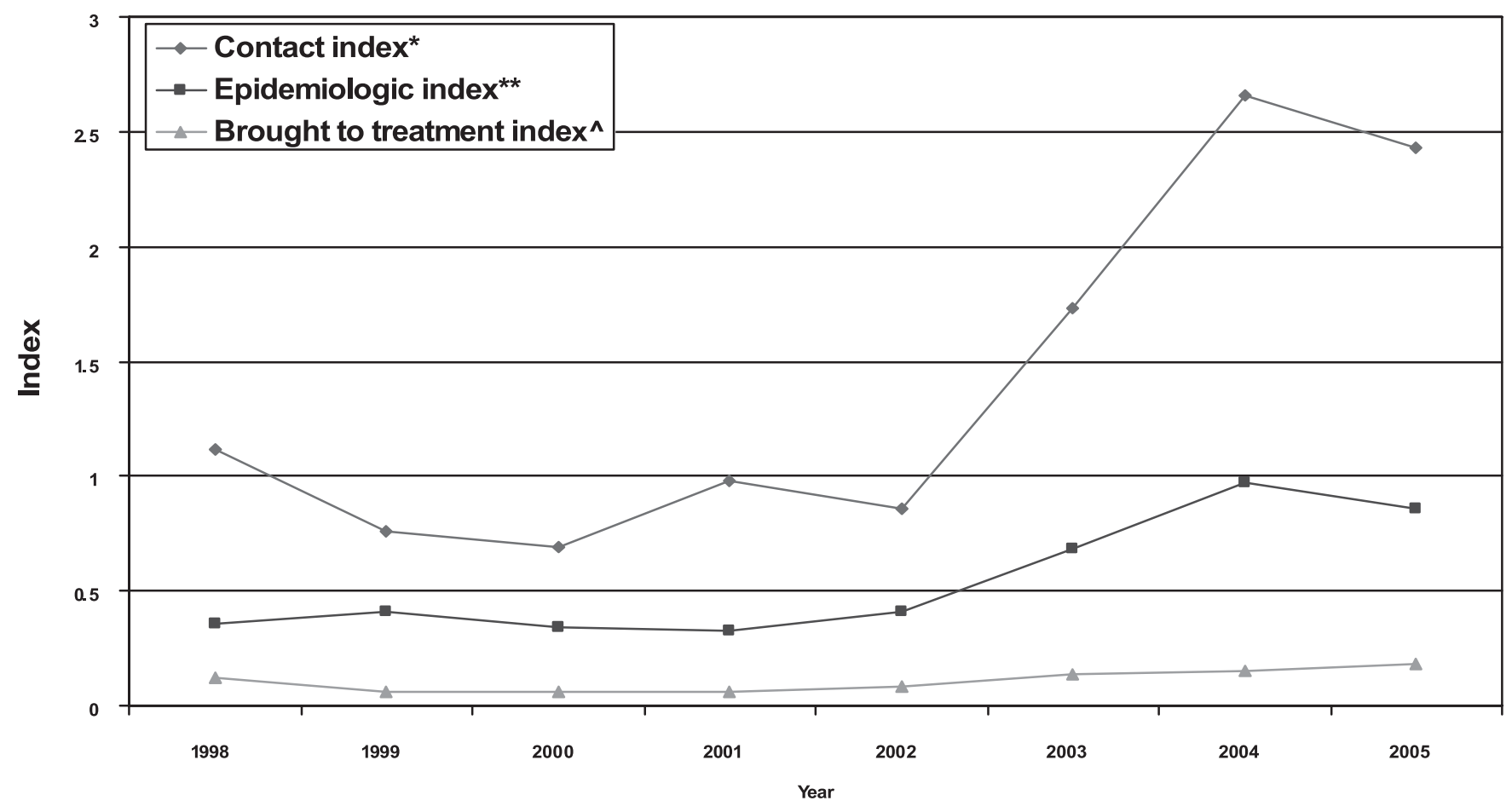

${ }^{*}$ Contact index $=$ Total contacts elicited/cases interviewed

${ }^{* *}$ Epidemiologic index $=$ Contacts preventively treated/cases interviewed

$\wedge$ Brought to treatment index=New cases identified and treated/cases interviewed

Fig. 3. Partner management indices related to early syphilis investigations King County, Washington, 1998-2005. 


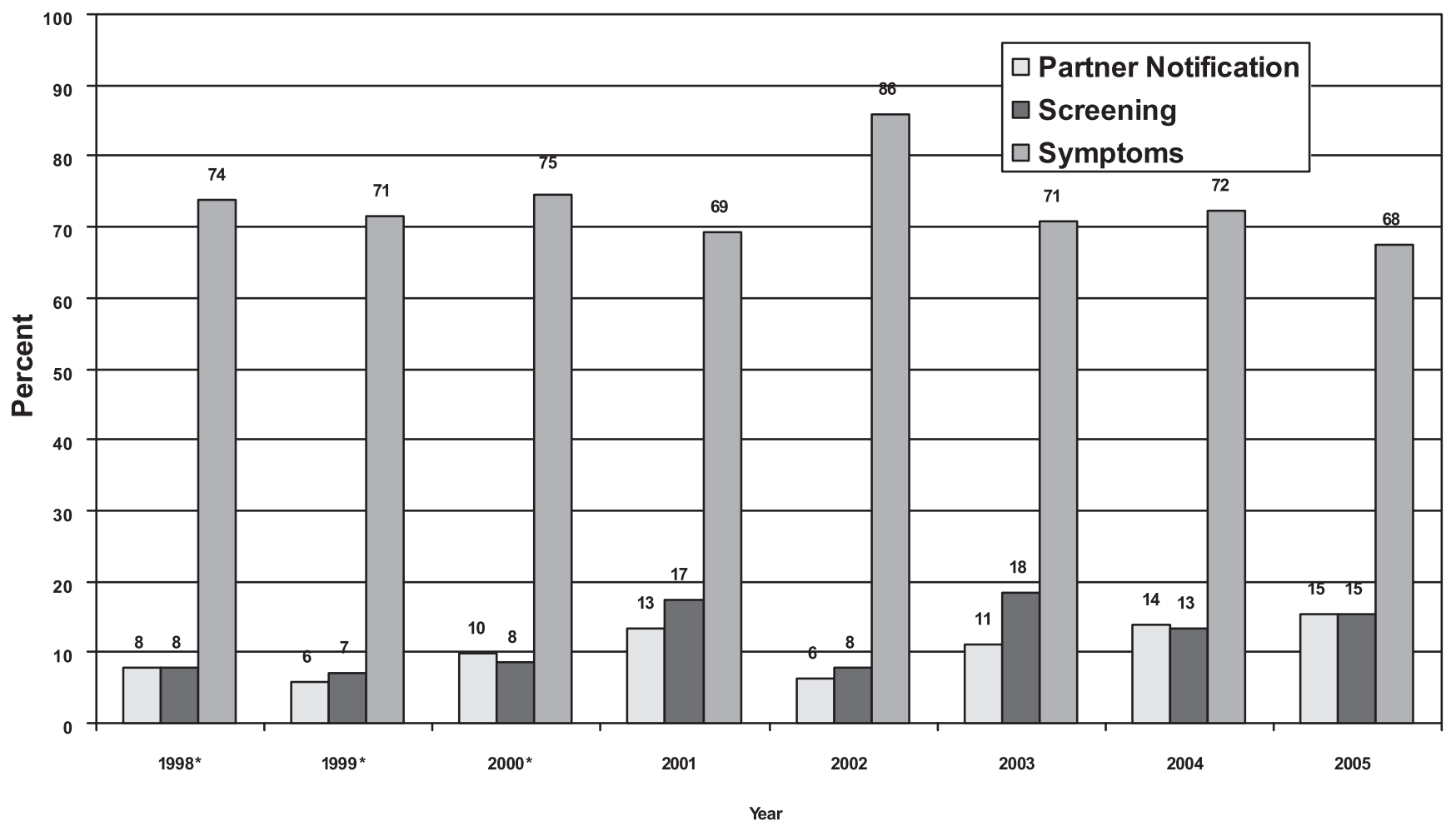

\section{*Percents do not add to 100 for $1998,1999,2000$, and 2005, as some cases for each of these years were missing information regarding the method of case detection}

Fig. 4. Percent of early syphilis cases diagnosed through partner notification, screening, and symptoms, King County, Washington, $1998-2005$.

index, epidemiologic index, and brought-to-treatment index remained relatively stable during the first several years of the MSM epidemic, when incidence was fairly stable. As the incidence of early syphilis rose from 2002 to 2005, the contact and epidemiologic indices increased through 2004, but the brought-to-treatment index did not change substantially. The percentage of all syphilis cases identified through partner notification activities rose from $8 \%$ in the period 1998 to 2002 to $14 \%$ in 2003 to 2005 . Twentyseven percent of women with early syphilis were identified through partner notification activities compared with $10 \%$ of heterosexual men and $12 \%$ of MSM.

Figure 4 displays the percentage of early syphilis cases detected through partner notification, serologic screening, and presentation with clinical evidence of syphilis. Of the 731 total cases, 527 $(71 \%)$ were detected because the patients presented with clinical evidence of syphilis; this percentage ranged from $69 \%$ to $86 \%$ annually over the study period. Although the proportion of cases diagnosed through screening and partner notification increased somewhat between 1998 and 2005, these increases were small with substantial variability over time.

To determine if any characteristics or behaviors were predictive of being diagnosed at later stages of infection, and therefore associated with a longer period of infectiousness, we conducted bivariate analyses of stage of disease and several behavioral and demographic characteristics among MSM for the period 2003 to 2005. The only statistically significant predictors of stage of diagnosis were HIV status and use of the Internet to recruit sex partners. During this time $17 \%$ of HIV-positive MSM were diagnosed with primary or secondary syphilis compared with $36 \%$ of
HIV-negative/status unknown men $(P<0.02)$. Among those who did not report Internet use to recruit sex partners, $76 \%$ were diagnosed with primary or secondary syphilis compared with $64 \%$ among Internet users $(P<0.01)$. Age, race, reported anonymous sex partners during the infectious period, drug use, and bathhouse attendance were not associated with disease stage. Data on anal sexual repertoire were not consistently available and consequently could not be analyzed.

To further examine Internet use and bathhouse attendance, we analyzed trends in these 2 activities among MSM for the period 2002 to 2005. Although the percentage of men reporting bathhouse attendance during this time decreased from $42 \%$ to $29 \%$, the percentage of men who reported using the Internet to recruit sex partners increased from $16 \%$ to $38 \%$ (Fig. 5).

\section{Discussion}

In recent years, outbreaks of syphilis among MSM have been reported from urban areas in many industrialized countries, ${ }^{9-17}$ and in 2005, an estimated $64 \%$ of all early syphilis cases in the United States occurred among MSM. ${ }^{18}$ The current epidemic of early syphilis among MSM in King County began in 1997 and continues unabated with incidence among MSM more than doubling between 2003 and 2005, and almost 2\% of all HIV-infected MSM being diagnosed with early syphilis annually. Additionally, syphilis reappeared in significant numbers in women and heterosexual men in 2004, although this epidemic abated in 2005. These trends occurred despite substantial expansion of 3 of the mainstays of traditional syphilis control in King County: serologic screening, 


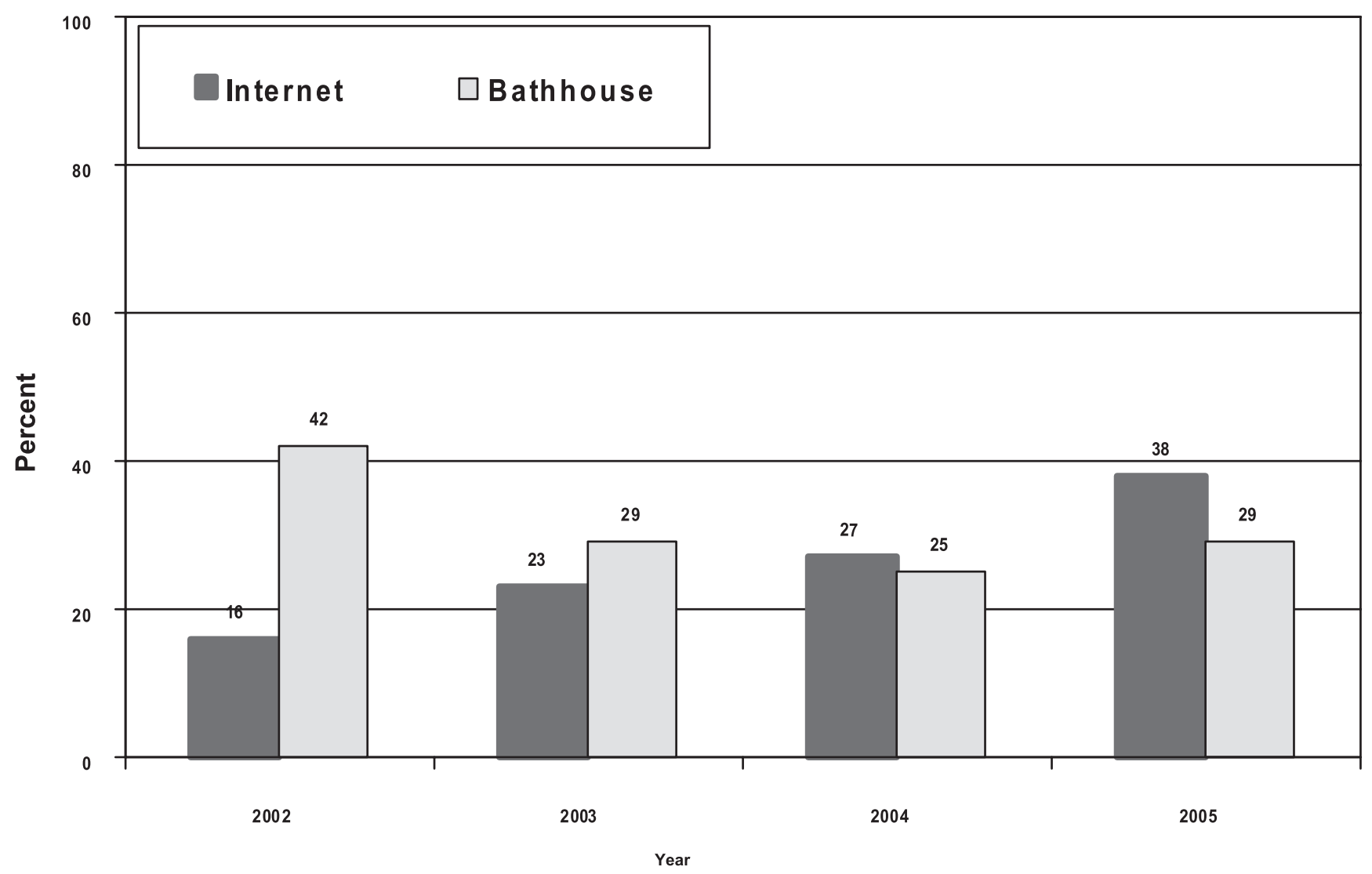

Fig. 5. Percentage of syphilis cases among men who have sex with men reporting Internet use to recruit sex partners or bathhouse attendance, 2002-2005.

partner notification activities, and outreach to the local MSM community through public awareness campaigns. Screening yields in our STD Clinic and outreach testing sites remained low, partner notification identified few cases, and the predominant means of identifying new syphilis cases continued to be through clinical evaluation of persons presenting with symptoms of syphilis.

Our findings are consistent with recent evaluations of syphilis control programs in other U.S. cities and expand on those findings by presenting trend data as well as information on how cases were identified. Cielsielski et al recently reported that the prevalence of early syphilis among over 14,000 MSM screened in 8 U.S. cities was $0.8 \%,{ }^{19}$ similar to our overall yield of $1.1 \%$ among over 37,000 tests performed. We observed a similarly low prevalence in outreach settings as well as a comparably low prevalence among STD clinic patients. Although it is possible that our program failed to identify a pool of latently infected MSM, given the large numbers of MSM tested, it seems more likely that such a pool does not exist in large numbers, even 8 years into our syphilis epidemic. To the extent that few cases of latent syphilis exist in our MSM population, our findings point to the success of our program in assuring that cases are identified and treated in a timely manner.

Our partner notification contact indices were similar to those reported by Hogben et $\mathrm{al}^{20}$ in a recent evaluation of syphilis partner notification programs in the same 8 U.S. cities studied by Cielsielski; these authors reported median contact and brought to treatment indices of 0.94 and 0.09 compared with our contact and brought-to-treatment indices of 1.73 and 0.14 , respectively, for 2003. Although Hogben did not report on trends, we observed increases in the contact and epidemiologic indices from 2002 to 2004 followed by slight declines in 2005. Despite these overall improvements, the number of cases identified through partner notification remains relatively low, and we have not observed a substantial change in our brought-to-treatment index.

We have conducted 3 public awareness campaigns since the MSM syphilis outbreak began. Although the campaigns have been well received, it is unclear whether they have influenced testing levels or the identification of new cases. In 2003, the percentage of King County early syphilis cases with primary or secondary syphilis was $73 \%$ compared with a range of $36 \%$ to $63 \%$ among 8 cities identified by the CDC as having high rates of MSM syphilis in 2003.21,22 The fact that the proportion of early cases with P\&S syphilis is higher locally than in other cities with MSM syphilis epidemics may indicate that either local syphilis awareness is high compared with other areas or that access to care is more widespread. Our random digit dial study of MSM found that in 2003, $53 \%$ of men believed STD were an increasing problem among MSM and that slightly over half of men with $>1$ sex partner in the preceding year had been tested for STD. The absence of similar data from earlier time periods or other cities makes them difficult to interpret. Studies of convenience samples of MSM in other cities have found roughly similar levels of testing. ${ }^{23}$ Regardless of how these findings compare with other cities, they suggest that further efforts to increase public awareness may be warranted. However, it is uncertain how meaningful a contribution public awareness efforts have had to controlling syphilis, particularly given the emphasis campaigns have often placed on screening and 
evidence suggesting that such testing may be of very limited efficacy in controlling the current epidemic. ${ }^{24}$

It is unclear why our efforts to control syphilis during this epidemic have been seemingly unsuccessful. Although the number of screening tests increased continuously during this time period, it may be that screening efforts have not reached MSM engaging in the most high-risk behaviors or that screening is simply not an effective method to find cases early in an epidemic when symptomatic disease is highly prevalent. Although the first public awareness campaign occurred 4 years after the epidemic began, rates rose dramatically in 2004 and 2005, after 3 consecutive years of our campaigns. The fact that many MSM with syphilis reported numerous anonymous partners certainly hampered our partner notification efforts. The problem of anonymous partners may have been amplified through an interaction between HIV serosorting, the practice of selectively engaging in unprotected sex with partners of the same HIV status, and the increasing use of the Internet to recruit sex partners. Finally, it is possible that our efforts actually curbed a more dramatic epidemic that would have occurred in the absence of our control efforts.

Our study has several limitations. Because a portion of our outreach screening for MSM is done anonymously (in conjunction with anonymous testing for HIV in outreach settings), we were unable to link individuals with positive screening results to actual diagnosed cases of early syphilis. Given this limitation, some of the screened individuals who had positive treponemal test results may not have been King County residents, and some may have had late rather than early syphilis. If late syphilis cases were included in our analyses of screening yield, the true yield of early syphilis cases from the screening venues we examined is even lower than we reported. Although we believe that most syphilis cases found through anonymous screening do seek care and then are reported as cases, anonymous testing does limit our ability to both track cases and evaluate our screening efforts. Additionally, our incidence figures for early syphilis among MSM may over- or underestimate the true incidence, because the size of the MSM population was calculated based on figures from a variety of studies carried out in several geographic areas. However, our current estimate of the proportion of men who are MSM in King County is similar to the proportion of men who reported themselves as homosexual or bisexual in the local 2003 to $2004 \mathrm{Be}$ havioral Risk Factor Surveillance System (Ann Glusker, personal communication). Additionally, even if our estimates were incorrect by a factor of 2, the incidence of syphilis among MSM in King County would still be extraordinarily high compared with other populations. Finally, ascertainment of Internet use to recruit sex partners and bathhouse attendance was not complete among our 2003-2005 cases. Responses were dichotomized as "yes" or "no/ unknown." Complete ascertainment of these behaviors would likely result in higher levels of both behaviors than we reported here.

The strengths of our study include a longstanding data collection and management system in the PHSKC STD Control Program. We also believe our data were relatively complete, because $91 \%$ of reported early syphilis cases during the study period were interviewed by PHSKC staff. Furthermore, laboratories as well as providers are required to report positive serologic tests for syphilis in Washington State, making underreporting of syphilis unlikely.

When the CDC announced its National Plan to Eliminate Syphilis in 1998, the stated goals of the program were to reduce the number of primary and secondary syphilis cases to less than 1,000 and to increase the percentage of syphilis-free counties to $90 \%$ by $2005 .{ }^{3}$ Although syphilis rates in heterosexuals continue to de- cline, the goal of eliminating syphilis is now jeopardized by an unforeseen international epidemic of STD among MSM, and CDC estimates that $64 \%$ of all early syphilis cases nationally now occur in MSM. ${ }^{18}$ Although some jurisdictions have implemented innovative programs to address the problem of syphilis in MSM, ${ }^{24}$ evaluations of these innovations have been very limited, and, to date, NPES has primarily emphasized improving and expanding traditional public health measures to control STD. Syphilis elimination programs using these measures may have successfully prevented an even larger MSM syphilis epidemic from occurring. However, our data, as well as those from other U.S. cities, suggest that the current approach is unlikely to eliminate syphilis and that a substantial midcourse correction in the NPES is needed. This correction should involve eliminating proscriptive aspects of the program and much greater emphasis on innovation, rigorous evaluation, and research, particularly in how to prevent syphilis in MSM.

\section{References}

1. Williams LA, Klausner JD, Whittington WL, Handsfield HH, Celum $\mathrm{C}$, Holmes KK. Elimination and reintroduction of primary and secondary syphilis. Am J Public Health 1999; 89:1093-1097.

2. Resurgent bacterial sexually transmitted disease among men who have sex with men-King County, Washington, 1997-1999. MMWR Morb Mortal Wkly Rep 1999; 48:773-777.

3. Centers for Disease Control and Prevention. The National Plan to Eliminate Syphilis From the United States. Atlanta: US Department of Health and Human Services, CDC, National Center for HIV, STD, and TB Prevention, October 1999.

4. Centers for Disease Control and Prevention. Sexually transmitted disease treatment guidelines. MMWR Morb Mortal Wkly Rep 2002; $51: 20$

5. Bibus DP, Wood RW, Hartfield K, Hanrahan M, Wood C. A model for distributing HIV-prevention resources. AIDS and Public Policy Journal 1994; 9:197-207.

6. Holmberg SD. The estimated prevalence and incidence of HIV in 96 large US metropolitan areas. Am J Public Health 1996; 86: 642-654.

7. HIV/AIDS Epidemiology Unit PH-SKC, and Infectious Disease and Reproductive Health Unit, Washington State Department of Health. HIV/AIDS Epidemiology Profile, December 1996.

8. Brewer DD, Golden MR, Handsfield HH. Unsafe sexual behavior and correlates of risk in a probability sample of men who have sex with men in the era of highly active antiretroviral therapy. Sex Transm Dis 2006; 33(4):250-255.

9. Primary and secondary syphilis among men who have sex with menNew York City, 2001. MMWR Morb Mortal Wkly Rep 2002; 51:853856.

10. Trends in primary and secondary syphilis and HIV infections in men who have sex with men-San Francisco and Los Angeles, California, 19982002. MMWR Morb Mortal Wkly Rep 2004; 53:575-578.

11. D'Souza G, Lee JH, Paffel JM. Outbreak of syphilis among men who have sex with men in Houston, Texas. Sex Transm Dis 2003; 30:872-873.

12. Transmission of primary and secondary syphilis by oral sex-Chicago, Illinois, 1998-2002. MMWR Morb Mortal Wkly Rep 2004; 53: 966-968.

13. Halsos AM, Edgardh K. An outbreak of syphilis in Oslo. Int J STD AIDS 2002; 13:370-372.

14. Stolte IG, Dukers NH, de Wit JB, Fennema JS, Coutinho RA. Increase in sexually transmitted infections among homosexual men in Amsterdam in relation to HAART. Sex Transm Infect 2001; 77:184186.

15. Simms I, Fenton KA, Ashton M, et al. The re-emergence of syphilis in the United Kingdom: The new epidemic phases. Sex Transm Dis 2005; 32:220-226.

16. Crook PD, Paine TC, Davis M, Fenton KA. London-The next battleground for syphilis? Commun Dis Public Health 2002; 5:163164. 
17. Jin F, Prestage GP, Kippax SC, et al. Epidemic syphilis among homosexually active men in Sydney. Med J Aust 2005; 183:179183.

18. Primary and secondary syphilis-United States, 2003-2004. MMWR Morb Mortal Wkly Rep 2006; 55:269-273.

19. Ciesielski C, Kahn RH, Taylor M, Gallagher K, Prescott LJ, Arrowsmith S. Control of syphilis outbreaks in men who have sex with men: The role of screening in nonmedical settings. Sex Transm Dis 2005; 32(suppl):S37-S42.

20. Hogben M, Paffel J, Broussard D, et al. Syphilis partner notification with men who have sex with men: A review and commentary. Sex Transm Dis 2005; 32(suppl):S43-S47.
21. Centers for Disease Control and Prevention. Sexually Transmitted Disease Surveillance, 2003. Atlanta: US Department of Health and Social Services, September 2004.

22. Peterman TA, Collins DE, Aral SO. Responding to the epidemics of syphilis among men who have sex with men: Introduction to the special issue. Sex Transm Dis 2005; 32(suppl):S1-3.

23. Vega MY, Roland EL. Social marketing techniques for public health communication: A review of syphilis awareness campaigns in 8 US cities. Sex Transm Dis 2005; 32(suppl):S30-S36.

24. Klausner JD, Kent CK, Wong W, McCright J, Katz MH. The public health response to epidemic syphilis, San Francisco, 1999-2004. Sex Transm Dis 2005; 32(suppl):S11-S18. 